\title{
Successful Painful Treatment of the Painful Achilles Was the Start for Research Providing New Knowledge about Tendon Pain and New Treatment Methods
}

\author{
Håkan Alfredson \\ Department of Surgical and Perioperative Science Sports Medicine, University of Umeå, Umeå, Sweden \\ Email: hakan.alfredsson@idrott.umea.se
}

Received 25 January 2014; revised 26 February 2014; accepted 2 April 2014

Copyright @ 2014 by author and Scientific Research Publishing Inc.

This work is licensed under the Creative Commons Attribution International License (CC BY). http://creativecommons.org/licenses/by/4.0/

(c) $\underset{\mathrm{EY}}{\mathrm{BY}}$ Open Access

\begin{abstract}
Successful treatment with painful eccentric training for patients with chronic painful midportion Achilles tendinosis started research on pain mechanisms involving ultrasound + Doppler investigations and immunohistochemical analyses of tendon tissue specimens. This research has resulted in a better understanding of the pain mechanisms involved in the chronic painful Achilles and patellar tendon, and based on these findings new non tendon-invasive treatment methods beneficial for the patients have been invented. This is a mini review showing the background and research that resulted in the highly successful mini surgical treatments for mid-portion Achilles tendinopathy and proximal patellar tendinopathy.
\end{abstract}

\section{Keywords}

Achilles, Patellar, Tendinopathy, Research, Ultrasound + Doppler, Eccentric Training, Sclerosing Injections, Surgical Scraping, Arthroscopic Shaving

\section{Introduction}

\subsection{Eccentric Calf Muscle Training}

By a co-incidence painful eccentric calf-muscle training was instituted on a group of patients with chronic painful midportion Achilles tendinopathy (Figure 1). This painful treatment model resulted in very good clinical results with pain-free and satisfied patients [1] [2], and also in larger patient groups [3] the results were good. 


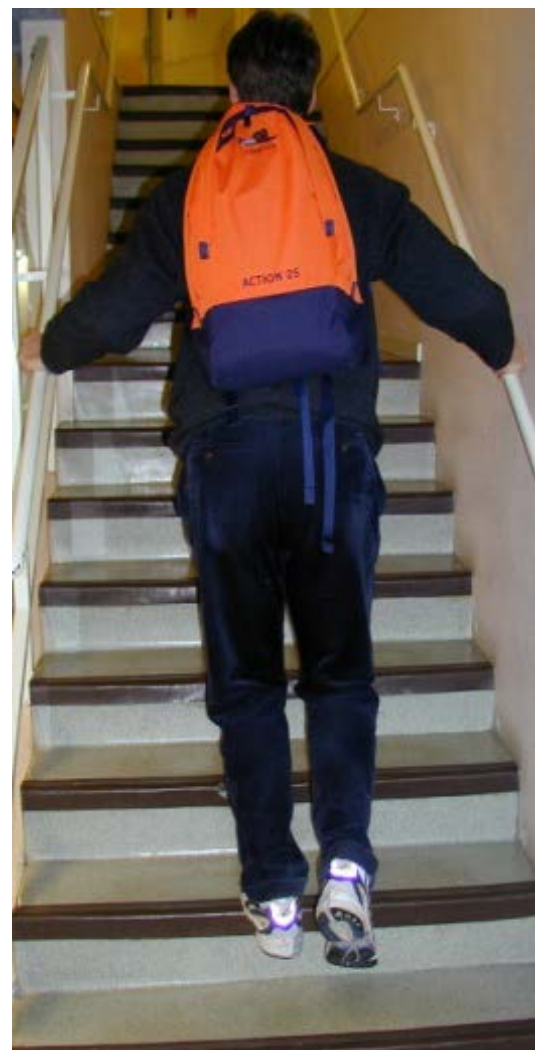

Figure 1. Eccentric calf muscle training.

Furthermore, ultrasound follow-ups showed improved and more normal tendon thickness and structure [4]. The findings were unexpected, and more or less against previous thinking on treatment of chronic painful tendons.

\subsection{Collaborative Research Project}

Consequently, to try to better understand the pain mechanisms involved in the chronic painful Achilles a collaborative research project involving sports medicine, radiology-ultrasound and anatomy, was started.

The first interesting findings were that ultrasound + Doppler examinations of chronic painful midportion Achilles tendinosis tendons showed high blood flow inside and outside ventral side (Figure 2), but in normal tendons there was no high blood flow [5].

This finding led to ultrasound + Doppler-guided biopsies taken from the region with high blood flow inside and outside the Achilles midportion. Immunohistochemical analyses of these biopsies showed nerves in close relation to blood vessels outside the tendon (Figure 3), but very few nerves inside the tendon [6]. There were mainly sympathetic, but also sensory, nerves [7]. Following ultrasound + Doppler-guided injections of small volumes of the local anaestetic xylocain + Adrenaline, targeting the regions with high blood flow outside the tendon, temporarily cured the tendon pain [6]. There were similar findings for the proximal patellar tendon in patients suffering from Jumper's knee [8].

\section{Invention of Treatment Methods}

\subsection{Ultrasound and Doppler-Guided Sclerosing Polidocanol Injections}

Based on the findings above, a new treatment method was invented. Ultrasound + Doppler-guided injections (Figure 4) of the sclerosing substance polidocanol, targeting the regions with high blood flow outside the tendon, was instituted [9]. This relatively technically demanding procedure showed good clinical results in pilot studies and also in a randomized placebo-controlled study [9] [10]. Interestingly, ultrasound + Doppler follow-ups showed decreased tendon thickness and improved structure over time [11], indicating a high potential in the soft 


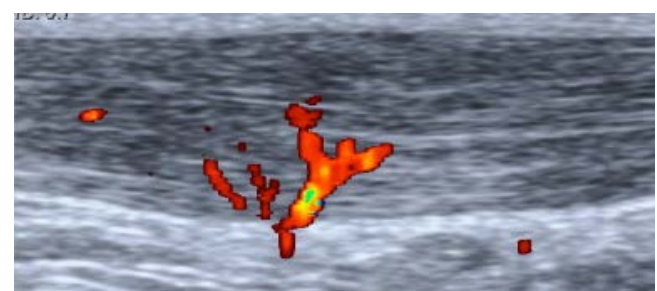

Figure 2. High blood flow (coloured structures) outside and inside the painful Achilles midportion.

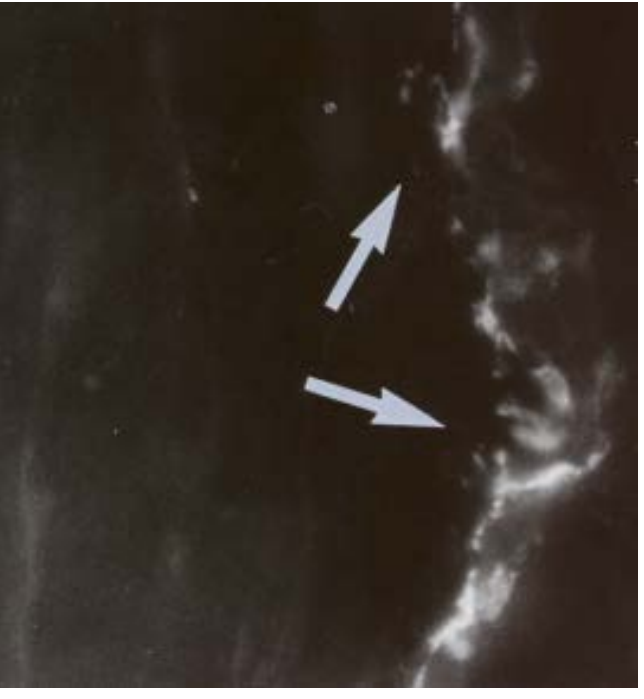

Figure 3. Biopsy from tendinosis patient, showing nerve structures (white) in close relation to blood vessel outside the tendon.

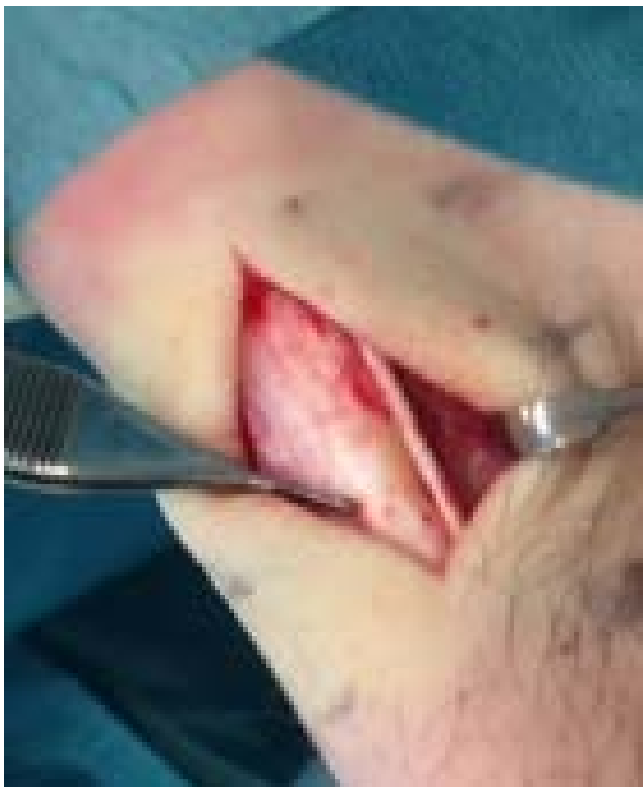

Figure 4. Plantaris tendon found in close relation to the medial side of the thickened Achilles tendon in a patient operated with a scraping procedure for midportion Achilles tendinosis. 
tissues outside the ventral side of the Achilles tendon. Also for the proximal patellar tendon (Jumper's knee) ultrasound + Doppler-guided sclerosing polidocanol injections showed good clinical results [12] [13]. The limitations with this type of treatment are that it is technically demanding, having a relatively long learning curve, and that most often multiple (Achilles 2 - 3 and patellar 4 - 5) injection treatments are needed for a good clinical result.

\subsection{Ultrasound and Doppler-Guided Mini-Surgical Scraping}

Because of the limitations noticed with sclerosing polidocanol injection treatment, instead a mini surgical scraping treatment was invented. Guided by the ultrasound + Doppler findings, in local anaestesia, a minor surgical procedure were the ventral side of the tendon was scraped in the regions with high blood flow and nerves, was performed (Figure 4) [14]. This is a one stage and more radical approach compared to the polidocanol injection treatment. Because there is no intra-tendinous trauma, a relatively fast (4 - 6 weeks) rehabilitation can be used postoperatively. This method started to be used on small numbers of non active and recreationally active individuals in 2007 [14], and because the clinical results were good without any side effects, the use of this method has been increased to large numbers of patients also including professional athletes [15]. Interestingly, the sometimes very nearby located plantaris tendon (Figure 4) not seldom seems to be involved in the painful condition, and consequently the surgical technique has been modified to always use a medial approach in order to evaluate a possible interference between the plantaris and Achilles tendons [16] [17].

\subsection{Ultrasound and Doppler-Guided Arthroscopic Shaving}

For the proximal patellar tendon (Jumper's knee), an ultrasound + Doppler-guided arthroscopic shaving procedure was invented (Figure 5), and the clinical results are similarly good as for the Achilles midportion [18] [19]. Ultrasound + Doppler follow-ups show decreased tendon thickness and a more normal structure over time (submitted).

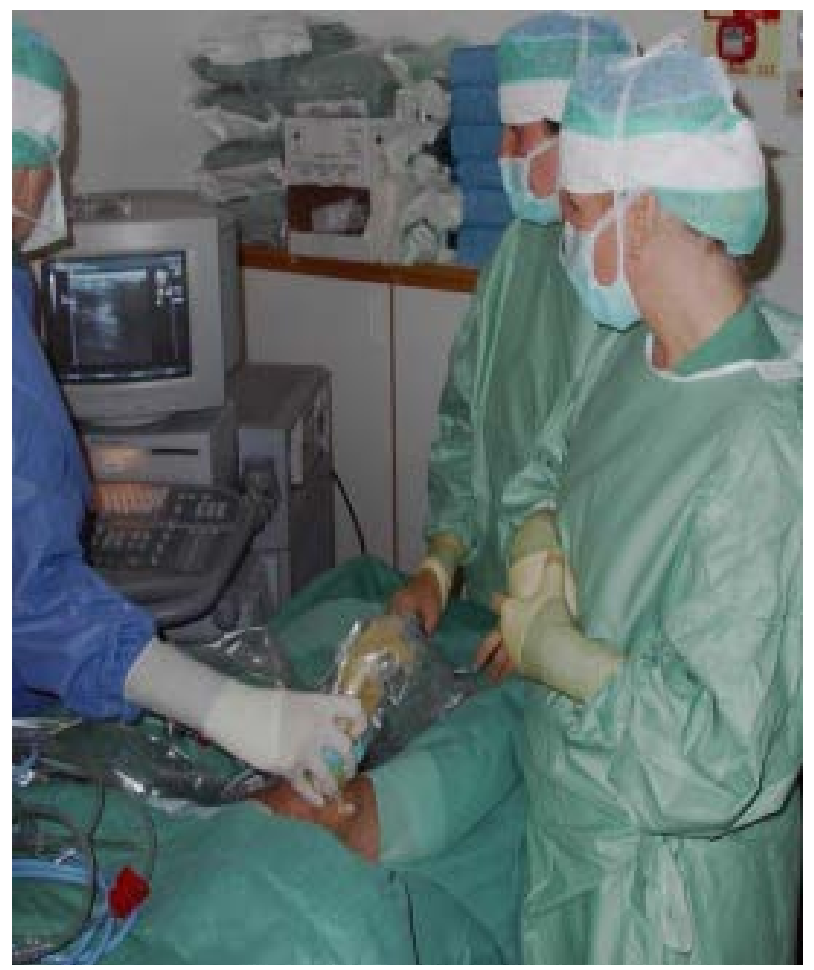

Figure 5. Ultrasound and Doppler-guided arthroscopic shaving in a patient with proximal patellar tendinopathy (Jumper's knee). The ultrasound picture (see monitor) is guiding the arthroscopic shaving procedure. 


\section{Implications for Treatment}

What have we learned about treatment from the research findings above? Well, before the chronic painful Achilles and patellar tendon, not responding to conservative treatment, was treated surgically [20] [21]. Surgical treatment most often included excision of macroscopically abnormal tendon tissue via a longitudinal tenotomy, followed by immobilization in a cast and often altogether 4 - 6 months of rehabilitation [20] [21]. This was because it was believed that to cure the pain treatment needed to be addressed to the inside of the tendon. The clinical results after intra-tendinous treatments has been demonstrated to be varying [22], and no improvement of tendon thickness and structure over time has been shown [23]. Now we know that the nerves associated with this chronic painful condition are located outside the tendon, and treatment/interference with these nerves outside the tendon can cure the pain and remodel the tendon over time. Also, there is no need for a long and careful rehabilitation after treatment/interference outside the tendon.

\section{Research on Tendon Cells}

In parallel with the research having a direct clinical application, research on the tendon cells has been performed. The hypercellularity seen in tendinosis tissue has previously been thought to represent tenocytes and tenoblasts [24]. However, looking close at those cells, not all have the traditional thin and slender shape characterizing a tenocyte, but instead there is a variety of cells with different shape. Our research group decided to have a closer look at these cells. Multiple tendon tissue biopsies, taken with ultrasound and Doppler-guidance from regions with irregular tendon structure and high blood flow, were analyzed with immune-histochemical and in-situ DNA-hybridazing techniques. Interestingly, some of these cells, were shown to produce pain and signal substances [25]-[30]. Also, the receptors for those substances were found to be localized on the cell surface [29] [30]. We are now only in the beginning of characterizing the cells in the hypercellular region of painful tendinosis tendons, but already we can state that there is a production of pain/signal substances and neurotransmitters inside the tendon. There are very few nerves inside the tendon, but instead there are multiple nerves outside the deep side of the tendon. It can be speculated whether there is a more or less cordless communication between the substances produced inside the tendon and the nerves located on the deep side of the tendinosis tendon? Hopefully, future research can clarify why the tendon cells start to produce these substances? If it is related to tendon pain? If it is related to structural tendon changes and thickening? And how this production can be blocked?

\section{Conclusions and Future Directions}

-In chronic painful tendinopathy/tendinosis in the Achilles midportion and proximal patellar tendon the nerves are located in close relation to blood vessels outside the deep side of the tendon.

-Ultrasound and Doppler examination can be used to localize the region with high blood flow (were the nerves are) outside the tendon, and to guide treatment procedures.

-Treatment with sclerosing polidocanol injections and mini surgical scraping, interfering with the regions with high blood flow and nerves outside the tendon, has shown good clinical results. The methods are safe with few complications, and there is a fast recovery and return to heavy tendon loading activity. In the longer perspective, remodeling decreased thickness and improved structure of the tendon.

-In my view there is no indication to use tenotomy in the treatment of patients with pure tendinopathy/tendinosis in the Achilles midportion and proximal patellar tendon.

-For surgical treatment of patients with the combination of tendinopathy/tendinosis and a partial rupture, tenotomy is sometimes needed.

-More research is needed to study the tendon cells. Is the production of pain and signal substances in the tendon cells of importance for the tendinopathy? Is it a trigger or a result? Studies clarify if blocking the production of pain and signal substances in the tendon cells has an impact on tendon structure and thickness, and if there is an influence on pain.

\section{References}

[1] Alfredson, H., Pietilä, T., Jonsson, P. and Lorentzon, R. (1998) Heavy-Loaded Eccentric Calf-Muscle Training for the 
Treatment of Chronic Achilles Tendinosis. American Journal of Sports Medicine, 26, 360-366.

[2] Mafi, N., Lorentzon, R. and Alfredson, H. (2001) Superior Results with Eccentric Calf-Muscle Training Compared to Concentric Training in a Randomized Prospective Multi-Center Study on Patients with Chronic Achilles Tendinosis. Knee Surgery, Sports Traumatology, Arthroscopy, 9, 42-47. http://dx.doi.org/10.1007/s001670000148

[3] Fahlström, M., Jonsson, P., Lorentzon, R. and Alfredson, H. (2003) Chronic Achilles Tendon Pain Treated with Eccentric Calf-Muscle Training. Knee Surgery, Sports Traumatology, Arthroscopy, 11, 327-333. http://dx.doi.org/10.1007/s00167-003-0418-z

[4] Öhberg, L., Lorentzon, R. and Alfredson, H. (2004) Eccentric Training in Patients with Chronic Achilles TendinosisNormalized Tendon Structure and Decreased Thickness at Follow-Up. British Journal of Sports Medicine, 38, 8-11. http://dx.doi.org/10.1136/bjsm.2001.000284

[5] Öhberg, L., Lorentzon, R. and Alfredson, H. (2001) Neovsacularisation in Achilles Tendons with Painful Tendinosis but Not in Normal Tendons: An Ultrasonographic Investigation. Knee Surgery, Sports Traumatology, Arthroscopy, 9, 233-238. http://dx.doi.org/10.1007/s001670000189

[6] Alfredson, H., Öhberg, L. and Forsgren, S. (2003) Is Vasculo-Neural in Growth the Cause of Pain in Chronic Achilles Tendinosis?-An Investigation Using Ultrasonogrphy and Colour Doppler, Immunohistochemistry, and Diagnostic Injections. Knee Surgery, Sports Traumatology, Arthroscopy, 11, 334-338. http://dx.doi.org/10.1007/s00167-003-0391-6

[7] Andersson, G., Danielson, P., Alfredson, H. and Forsgren, S. (2007) Nerve-Related Characteristics of Ventral Paratendinous Tissue in Chronic Achilles Tendinosis. Knee Surgery, Sports Traumatology, Arthroscopy, 15, 1272-1279. http://dx.doi.org/10.1007/s00167-007-0364-2

[8] Danielsson, P., Andersson, G., Alfredson, H. and Forsgren, S. (2008) Marked Sympathetic Component in the Perivascular Innervation of the Dorsal Paratendinous Tissue of the Patellar Tendon in Arthroscopically Treated Tendinosis Patients. Knee Surgery, Sports Traumatology, Arthroscopy, 16, 621-626. http://dx.doi.org/10.1007/s00167-008-0530-1

[9] Öhberg, L. and Alfredson, H. (2002) Ultrasound Guided Sclerosing of Neovessels in Painful Chronic Achilles Tendinosis: Pilot Study of a New Treatment. British Journal of Sports Medicine, 36, 173-177. http://dx.doi.org/10.1136/bjsm.36.3.173

[10] Alfredson, H. and Öhberg, L. (2005) Sclerosing Injections to Areas of Neovascularisation Reduce Pain in Chronic Achilles Tendinopathy: A Double-Blind Randomized Controlled Trial. Knee Surgery, Sports Traumatology, Arthroscopy, 13, 338-344. http://dx.doi.org/10.1007/s00167-004-0585-6

[11] Lind, B., Öhberg, L. and Alfredson, H. (2006) Sclerosing Polidocanol Injections in Mid-Portion Achilles Tendinosis: Remaining Good Clinical Results and Decreased Tendon Thickness at 2-Year Follow-Up. Knee Surgery, Sports Traumatology, Arthroscopy, 14, 1327-1332. http://dx.doi.org/10.1007/s00167-006-0161-3

[12] Alfredson, H. and Öhberg, L. (2005) Neovascularisation in Chronic Painful Patellar Tendinosis Promising Results after Sclerosing Neovessels outside the Tendon Challenges the Need for Surgery. Knee Surgery, Sports Traumatology, Arthroscopy, 13, 74-80. http://dx.doi.org/10.1007/s00167-004-0549-x

[13] Hoksrud, A., Öhberg, L., Alfredson, H. and Bahr, R. (2006) Ultrasound-Guided Sclerosis of Neovessels in Painful Chronic Patellar Tendinopathy-A Randomized Controlled Trial. American Journal of Sports Medicine, 34, 17381746. http://dx.doi.org/10.1177/0363546506289168

[14] Alfredson, H., Öhberg, L., Zeisig, E. and Lorentzon, R. (2007) Treatment of Midportion Achilles Tendinosis: Similar Clinical Results with US and CD-Guided Surgery outside the Tendon and Sclerosing Polidocanol Injections. Knee Surgery, Sports Traumatology, Arthroscopy, 15, 1504-1509. http://dx.doi.org/10.1007/s00167-007-0415-8

[15] Alfredson, H. (2011) Ultrasound and Doppler-Guided Mini-Surgery to Treat Midportion Achilles Tendinosis: Results of a Large Material and a Randomised Study Comparing two Scraping Techniques. British Journal of Sports Medicine, 45, 407-410. http://dx.doi.org/10.1136/bjsm.2010.081216

[16] Alfredson, H. (2011) Midportion Achilles Tendinosis and the Plantaris Tendon. British Journal of Sports Medicine, 45, 1023-1025. http://dx.doi.org/10.1136/bjsports-2011-090217

[17] Spang, C., Alfredson, H., Ferguson, M., Roos, B., Bagge, J. and Forsgren, S. (2013) The Plantaris Tendon in Association with Midportion Achilles Tendinosis: Tendinosis-Like Morphological Features and Presence of a Non-Neuronal Cholinergic System. Histology and Histopathology, 28, 623-632.

[18] Willberg, L., Sunding, K., Forsblad, M. and Alfredson, H. (2007) Ultrasound- and Doppler-Guided Arthroscopic Shaving to Treat Jumper's Knee. Knee Surgery, Sports Traumatology, Arthroscopy, 15, 1400-1403. http://dx.doi.org/10.1007/s00167-007-0360-6

[19] Willberg, L., Sunding, K., Forssblad, M., Fahlstrom, M. and Alfredson, H. (2011) Sclerosing Polidocanol Injections or Arthroscopic Shaving to Treat Patellar Tendinopathy/Jumper's Knee? A Randomised Controlled Study. British Journal of Sports Medicine, 45, 411-415. http://dx.doi.org/10.1136/bjsm.2010.082446 
[20] Leadbetter, W.B., Mooar, P.A., Lane, G.J. and Lee, S.J. (1992) The Surgical Treatment of Tendinitis. Clinical Rationale and Biologic Basis. Clinics in Sports Medicine, 11, 679-712

[21] Nelen, G., Martens, M. and Burssens, A. (1989) Surgical Treatment of Chronic Achilles Tendinitis. American Journal of Sports Medicine, 17, 754-759. http://dx.doi.org/10.1177/036354658901700605

[22] Tallon, C., Coleman, B.D., Khan, K.M. and Maffulli, N. (2001) Outcome of Surgery for Chronic Achilles Tendinopathy. A Critical Review. American Journal of Sports Medicine, 29, 315-320.

[23] Alfredson, H., Zeisig, E. and Fahlström, M. (2009) No Normalisation of the Tendon Structure and Thickness after IntraTendinous Surgery for Chronic Painful Midportion Achilles Tendinosis. British Journal of Sports Medicine, 43, 948949. http://dx.doi.org/10.1136/bjsm.2008.050955

[24] Åström, M. (1997) On the Nature and Etiology of Chronic Achilles Tendinopathy. Dissertation, University of Lund, Lund.

[25] Danielsson, P., Alfredson, H. and Forsgren, S. (2006) Immunohistochemical and Histochemical Findings Favoring the Occurrence of Autocrine/Paracrine as Well as Nerve-Related Cholinergic Effects in Chronic Painful Patellar Tendon Tendinosis. Microscopy Research and Technique, 69, 808-819. http://dx.doi.org/10.1002/jemt.20351

[26] Danielson, P., Alfredson, H. and Forsgren, S. (2007) Studies on the Importance of Sympathetic Innervation, Adrenergic Receptors, and a Possible Local Catecholamine Production in the Development of Patellar Tendinopathy (Tendinosis) in Man. Microscopy Research and Technique, 80, 2235-2238.

[27] Danielson, P., Andersson, G., Alfredson, H. and Forsgren, S. (2007) In Situ Hybridization Studies Confirming Recent Findings of the Existence of a Local Non-Neuronal Catecholamine Production in Human Patellar Tendinosis. Microscopy Research and Technique, 70, 908-911. http://dx.doi.org/10.1002/jemt.20495

[28] Bjur, D., Danielsson, P., Alfredson, H. and Forsgren, S. (2008) Immunohistochemical and in Situ Hybridization Observations Favour a Local Catecholamine Production in the Human Achilles Tendon. Histology and Histopathology, 23, 197-208.

[29] Bjur, D., Danielson, P., Alfredson, H. and Forsgren, S. (2008) Presence of a Non-Neuronal Cholinergic System and Occurrence of Up- and Down Regulation in Expression of $\mathrm{M}_{2}$ Muscarinic Acetylcholine Receptors: New Aspects of Importance Regarding Achilles Tendon Tendinosis (Tendinopathy). Cell and Tissue Research, 331, 385-400. http://dx.doi.org/10.1007/s00441-007-0524-1

[30] Andersson, G., Danielsson, P., Alfredson, H. and Forsgren, S. (2008) Presence of Substance P and the Neurokinin-1 Receptor in Tenocytes of the Human Achilles Tendon. Regulatory Peptides, 150, 81-87. http://dx.doi.org/10.1016/j.regpep.2008.02.005 